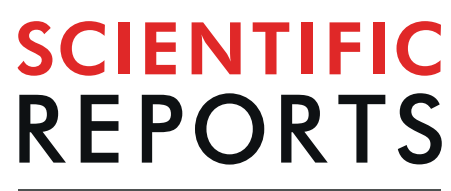

natureresearch

\title{
The role of magnetic resonance imaging in the preoperative evaluation of anal fistulas
}

\author{
Duc Vo ${ }^{1 *}$, Chien Phan ${ }^{1}$, Linh Nguyen ${ }^{1}$, Huyen Le ${ }^{1}$, Tin Nguyen ${ }^{2}$ \& Hung Pham ${ }^{1,3}$
}

This study aimed to determine the role of magnetic resonance imaging (MRI) in diagnosing and describing the characteristics of fistula-in-ano, and the agreement between MRI and operative findings. We conducted a retrospective study in 367 patients with fistula-in-ano who were diagnosed and had an operation at the University Medical Center between January 2016 and January 2018. MRI findings were evaluated and compared with surgical findings using the kappa coefficient (k) method. 367 patients ( 327 male and 40 female, mean age $39.3 \pm 12.4$ years). A total of 411 primary fistulas were found during surgery. There was a strong agreement between MRI and surgery for classifying primary tracts $(k=0.89)$ and detecting secondary tracts $(k=0.94)$. While the sensitivity and specificity of MRI for detecting internal openings were $99 \%$ and $85.2 \%$ respectively; these rates were $100 \%$ for abscesses. Both T2-weighted turbo spin-echo (T2W TSE) and postcontrast fat-saturated T1-weighted turbo spinecho (FS T1W TSE) sequences showed high sensitivity $(96.6 \%$ and $98.4 \%$ respectively) and specificity (92.6\% and $81.5 \%$ respectively) for depicting internal openings and secondary tracts. Post-contrast FS T1W TSE sequence was very effective in detecting abscesses with an accuracy of $100 \%$. In conclusion, MRI can be considered an accurate tool for the preoperative evaluation of fistula-in-ano, which is a major determinant of the surgical outcome. Both T2W TSE and post-contrast FS T1W TSE sequences are highly accurate in depicting the features of fistula-in-ano. If there are no contraindications, contrast administration is recommended to differentiate abscesses from active inflammation.

Fistula-in-ano is an inflammatory disorder of anorectal region characterised by a tract between the anal canal and the perianal skin ${ }^{1,2}$. Fistula-in-ano is usually a sequela of a poorly managed perianal abscess. This condition can also be associated with tuberculosis, cancer, and radiotherapy, etc. ${ }^{2,3}$.

Fistula-in-ano is the second most common anorectal disease after haemorrhoids ${ }^{2}$. Surgery is considered the treatment of choice aiming to avoid recurrence and preserve anal sphincter function. The risk of recurrence increases to $25 \%$ if surgeons fail to recognise and remove radically a fistula and its associated elements during corrective surgery, especially internal openings and secondary tracts ${ }^{1,4-6}$. Accordingly, a precise and comprehensive preoperative assessment of fistula tract is a pivotal diagnostic strategy and contributes significantly to the success rate of surgery.

Before the era of magnetic resonance imaging (MRI), fistulography was used to evaluate fistula-in-ano. However, this technique has a low diagnostic accuracy $(\sim 16 \%)$, and inability to visualise secondary tracts, abscesses and the sphincter complex due to its suboptimal contrast opacification ${ }^{7}$. As a result, fistulograms are not able to provide information about the relationship between fistula tracts and anal sphincters. Endoanal ultrasonography is the first imaging technique that provides the anatomical details of anal canal ${ }^{1}$. It can be used for the diagnosis and management of not only abscesses and fistula-in-ano, but also anorectal and prostate tumours. Endoanal ultrasonography is particularly helpful in identifying primary fistulous tracts and internal openings with high accuracy rates ${ }^{8,9}$. However, the limited field of view is regarded as an inherent limitation of this technique, discounting its value to evaluate secondary tracts or supralevator extensions of a primary tract.

Recently, MRI has been considered the 'gold standard' technique for the preoperative evaluation of fistula-in-ano. An accurate and comprehensive assessment to detect primary tracks, associated ramifications and abscesses plays a crucial role in determining surgical outcomes and minimising complications, such as faecal incontinence, as well as recurrent lesions ${ }^{1,10-12}$.

${ }^{1}$ Department of Diagnostic Imaging, University Medical Center, Ho Chi Minh City, Vietnam. ${ }^{2}$ Department of Proctology, University Medical Center, Ho Chi Minh City, Vietnam. ${ }^{3}$ Adelaide Medical School, The University of Adelaide, Adelaide, SA, Australia. *email: duc.vt@umc.edu.vn 


\begin{tabular}{|c|c|c|c|c|c|c|c|c|}
\hline \multicolumn{2}{|l|}{ Sequence } & \begin{tabular}{|l|} 
TR \\
$(\mathrm{ms})$
\end{tabular} & \begin{tabular}{|l|} 
TE \\
$(\mathbf{m s})$
\end{tabular} & $\begin{array}{l}\text { FOV } \\
(\mathrm{cm})\end{array}$ & Matrix & $\begin{array}{l}\text { Thick } \\
(\mathrm{mm})\end{array}$ & $\begin{array}{l}\text { Gap } \\
(\mathbf{m m})\end{array}$ & NSA \\
\hline \multicolumn{2}{|l|}{ T2W TSE sagittal } & 4570 & 86 & 23 & $320 \times 256$ & 3,5 & 0,35 & 1 \\
\hline \multicolumn{2}{|l|}{ T2W TSE axial } & 5000 & 86 & 20 & $320 \times 240$ & 3,5 & 0,35 & 2 \\
\hline \multicolumn{2}{|l|}{ FS T2W TSE axial } & 5160 & 86 & 20 & $320 \times 240$ & 3,5 & 0,35 & 2 \\
\hline \multicolumn{2}{|l|}{ FS T2W TSE coronal } & 3220 & 74 & 25 & $320 \times 240$ & 3,2 & 0,32 & 1 \\
\hline \multicolumn{2}{|l|}{ T1W TSE axial } & 544 & 10 & 20 & $320 \times 224$ & 3,5 & 0,35 & 1 \\
\hline \multicolumn{2}{|l|}{ FS T1W TSE axial } & 670 & 10 & 20 & $320 \times 224$ & 3,5 & 0,35 & 1 \\
\hline \multirow{3}{*}{ Postcontrast FS T1W TSE } & axial & 670 & 10 & 20 & $320 \times 224$ & 3,5 & 0,35 & 2 \\
\hline & coronal & 600 & 12 & 25 & $320 \times 256$ & 3,2 & 0,32 & 1 \\
\hline & sagittal & 655 & 12 & 23 & $320 \times 224$ & 3,5 & 0,35 & 1 \\
\hline
\end{tabular}

Table 1. MRI sequences for preoperative assessment of fistula-in-ano. TSE: turbo spin echo, FS: fat-saturated, TR: repetition time, TE: echo time, FOV: field of view, NSA: number of acquisitions.

The aim of this study was to evaluate the accuracy of MRI for the diagnosis and characterisation of fistula-in-ano and the agreement between preoperative MRI and surgical findings.

\section{Materials and Methods}

This retrospective study was conducted in the University Medical Center, Ho Chi Minh City. The protocol was reviewed and approved by the Human Research Ethics Committee of the University Medical Center of Ho Chi Minh City. The study was performed in accordance with the Declaration of Helsinki. All the patients, or their guardians, provided written informed consent prior to their involvement in the study.

Subjects. The images of patients who had preoperative MRI assessment and surgery for fistula-in-ano from January 12016 to January 312018 were collected. All patients also underwent a physical examination by a proctologist to document the number and location of cutaneous openings after detailed medical history had been collected.

MRI protocol. MRI examinations were performed on either 1.5 T (Magnetom Avanto, Siemens Healthcare Limited, Germany) or 3.0 T MR (Magnetom, Siemens Healthcare Limited, Germany) scanners using a phased-array surface coil with 6 channels.

No patient preparation was required and patients were placed flat on their back. Precontrast images obtained were as follows: sagittal T2-weighted turbo spin-echo (T2W TSE), oblique coronal fat-saturated (FS) T2W TSE, oblique axial T2W TSE, oblique axial FS T2W TSE, oblique axial T1-weighted turbo spin-echo (T1W TSE) and axial FS T1W TSE. After an intravenous administration of gadolinium $(0.2 \mathrm{ml} / \mathrm{kg})$, post-contrast FS T1W TSE images were acquired in three planes (sagittal, coronal and axial). Parameters of the used sequences are shown in Table 1.

Image analysis. Images were interpreted and reported independently on picture archiving and communication system (PACS) by two radiologists who had more than 5 years of experience in analysing fistula-in-ano MRI. In the cases where there were discrepancies in interpretation between the two radiologists, a senior radiologist's evaluation was considered the final result.

The following characteristics were assessed for each fistula-in-ano: the location of primary tracts, the presence of secondary tracts and abscess formation and the site of internal and external openings. Fistulas were classified according to the Parks and St. James's University Hospital classifications ${ }^{13,14}$. In the image interpretation, it was assumed that a fluid collection larger than $10 \mathrm{~mm}$ in diameter with rim enhancement on post-contrast $\mathrm{T} 1 \mathrm{~W}$ TSE images was an abscess as per the criteria of Singh et al. and Torkzad et al. ${ }^{15,16}$.

During surgery, the characteristics of each fistula-in-ano were also carefully documented and then used as a reference standard to compare to MRI findings.

Statistical analysis. For each MRI characteristic, $2 \times 2$ contingency table was used to calculate sensitivity, specificity, positive predictive value (PPV), negative predictive value (NPV), and diagnostic accuracy. Agreement between the MRI and surgical findings was assessed using the weighted kappa coefficient (k) with a 95\% confidence interval. The degree of agreement was classified as follows: poor $(\mathrm{k}<0.2)$, fair $(0.2 \leq \mathrm{k}<0.4)$, moderate $(0.4 \leq \mathrm{k}<0.6)$, good $(0.6 \leq \mathrm{k}<0.8)$, or very good $(\mathrm{k} \geq 0.8)$. All analyses were performed using STATA version 14 (STATA Corp., Texas, USA). A P value $<0.05$ was considered significant in all analyses. Data are shown as mean values \pm SDs.

\section{Results}

367 patients were eligible for the study. They were 320 males and 47 females with a ratio of 9:1. Their ages ranged from 12 to 84 years with a mean of $39.3 \pm 12.4$ years. Of 367 patients, $6.0 \%$ were aged over 60 years, $91.6 \%$ were aged from 20 to 60 years and only $2.4 \%$ were aged less than 20 years. $27 \%$ of patients had previous surgery for anal fistula. 


\begin{tabular}{|l|l|l|l|}
\hline & $\leq \mathbf{3} \mathbf{c m}$ & $\mathbf{3}-\mathbf{5} \mathbf{c m}$ & $>\mathbf{5 c m}$ \\
\hline Intersphincteric fistula & $\begin{array}{l}88 \\
(95.7 \%)\end{array}$ & $\begin{array}{l}4 \\
(4.3 \%)\end{array}$ & $\begin{array}{l}0 \\
(0 \%)\end{array}$ \\
\hline Low transsphincteric fistula & $\begin{array}{l}150 \\
(71.8 \%)\end{array}$ & $\begin{array}{l}46 \\
(22.0 \%)\end{array}$ & $\begin{array}{l}13 \\
(6.2 \%)\end{array}$ \\
\hline High transsphincteric fistula & $\begin{array}{l}5 \\
(9.3 \%)\end{array}$ & $\begin{array}{l}45 \\
(83.3 \%)\end{array}$ & $\begin{array}{l}4 \\
(7.4 \%)\end{array}$ \\
\hline Supra and extrasphincteric fistula & $\begin{array}{l}0 \\
(0 \%)\end{array}$ & $\begin{array}{l}10 \\
(83.3 \%)\end{array}$ & $\begin{array}{l}2 \\
(16.7 \%)\end{array}$ \\
\hline
\end{tabular}

Table 2. Distance between external opening and anal verge.

\begin{tabular}{|l|l|l|l|l|l|l|l|l|}
\hline Surgery & Inter & Trans & Supra & Extra & Superficial & $\begin{array}{l}\text { Blind } \\
\text { tract }\end{array}$ & $\begin{array}{l}\text { No primary } \\
\text { tract }\end{array}$ & Total \\
\hline MRI & 92 & 16 & 0 & 0 & 0 & 4 & 1 & 113 \\
\hline Inter & 0 & 245 & 0 & 0 & 0 & 0 & 0 & 245 \\
\hline Trans & 0 & 0 & 3 & 0 & 0 & 0 & 0 & 3 \\
\hline Supra & 0 & 0 & 0 & 9 & 0 & 0 & 0 & 9 \\
\hline Extra & 0 & 0 & 0 & 0 & 7 & 0 & 0 & 7 \\
\hline Superficial & 0 & 0 & 0 & 0 & 0 & 32 & 0 & 32 \\
\hline Blind tract & 0 & 2 & 0 & 0 & 0 & 1 & 0 & 3 \\
\hline $\begin{array}{l}\text { No primary } \\
\text { tract }\end{array}$ & 0 & 263 & 3 & 9 & 7 & 37 & 1 & 412 \\
\hline Total & 92 & 263 & & & & \\
\hline
\end{tabular}

Table 3. Agreement between MRI and surgery in the classification of primary tract according to the Parks classification. Inter: Intersphincteric fistula, Trans: transsphincteric fistula, Supra: suprasphincteric fistula, Extra: extrasphincteric fistula, Superficial: superficial fistula. Data are presented as numbers of fistulas. Kappa value: $0.89(0.85-0.94), \mathrm{p}<0.001$.

\begin{tabular}{|l|l|}
\hline Grade & Number \\
\hline Grade 1 & $\begin{array}{l}89 \\
(24.1 \%)\end{array}$ \\
\hline Grade 2 & $\begin{array}{l}24 \\
(6.5 \%)\end{array}$ \\
\hline Grade 3 & $\begin{array}{l}157 \\
(42.4 \%)\end{array}$ \\
\hline Grade 4 & $\begin{array}{l}88 \\
(23.8 \%)\end{array}$ \\
\hline Grade 5 & $\begin{array}{l}12 \\
(3.2 \%)\end{array}$ \\
\hline
\end{tabular}

Table 4. MRI grading of anal fistulas according to St. James's University Hospital classification.

External opening. The external openings were identified in 353/367 patients (96.2\%) with 442 external openings. $289(81.9 \%)$ patients had one external opening. 64 (18.1\%) patients had multiple external openings: 47 (73.4\%) had two, $10(15.6 \%)$ had three, $6(9.4 \%)$ had four and $1(1.6 \%)$ had five external openings.

The mean distance between external opening and anal verge was $2.9 \pm 2 \mathrm{~cm}$. Distances of different fistula types are shown in Table $2.95 \%$ of external openings were within $5 \mathrm{~cm}$ from the anal verge and $60.4 \%$ of external openings were posterior.

Primary tract. 411 primary tracts were detected in 367 (\%) patients during surgery. $263(64.0 \%)$ tracts were transsphincteric, 92 (22.4\%) tracts were intersphincteric according to the Parks classification (Table 3). Using this classification system, surgeons also reported 7 (1.7\%) superficial fistulas and $37(9.0 \%)$ blind tracts, which were left unclassified. Up to $333(90.7 \%)$ patients had a single primary tract and $3(0.8 \%)$ patients had four primary tracts.

According to the St. James's University Hospital classification, 89 (24.1\%) patients had grade 1, 24 (6.5\%) patients had grade 2, $157(42.4 \%)$ patients had grade 3, $88(23.8 \%)$ patients had grade 4 , and $12(3.2 \%)$ patients had grade 5 fistulas (Table 4).

Low transsphincteric fistulas occurred more frequently (79.5\%) than high transsphincteric fistulas (20.5\%). There was a strong agreement for primary tract classification between MRI and surgical findings, with $\mathrm{k}=0.89$ (Table 3).

Internal opening. 385 internal openings were detected in 367 patients. 360 (93.5\%) internal openings were located at the dentate line with a mean distance to the anal verge of $2.2 \pm 0.2 \mathrm{~cm}$ and $179(46.5 \%)$ internal openings were located at 6 o'clock position. 


\begin{tabular}{|c|c|c|c|c|c|c|c|c|c|}
\hline MRI & Surgery & Perianal & Inter & Ischio & Supra & \begin{tabular}{|l|} 
Deep \\
postanal
\end{tabular} & Sub & $\begin{array}{l}\text { No secondary } \\
\text { tract }\end{array}$ & Total \\
\hline \multicolumn{2}{|c|}{ Perianal } & 3 & & & & & & & 3 \\
\hline \multicolumn{2}{|l|}{ Inter } & & 45 & & & & & 6 & 51 \\
\hline \multicolumn{2}{|c|}{ Ischio } & & & 56 & & & & 3 & 59 \\
\hline \multicolumn{2}{|l|}{ Supra } & & & & 14 & & & 2 & 16 \\
\hline \multicolumn{2}{|c|}{ Deep postanal } & & & & & 3 & & & 3 \\
\hline \multicolumn{2}{|l|}{ Sub } & & & & & & 0 & & 0 \\
\hline \multicolumn{2}{|c|}{ No secondary tract } & & 1 & & & & 1 & 266 & 268 \\
\hline \multicolumn{2}{|l|}{ Total } & 3 & 46 & 56 & 14 & 3 & 1 & 277 & 400 \\
\hline
\end{tabular}

Table 5. Agreement between MRI and surgery for detecting the location of secondary tracts. Perianal: perianal space, Inter: Intersphincteric space, Ischio: ischioanal space, Supra: supralevator space, Deep postanal: deep postanal space, Sub: submucosa space. Data are presented as numbers of secondary tracts. Kappa value: 0.94 (0.90-0.97), $\mathrm{p}<0.001$.

\begin{tabular}{|l|l|l|l|l|l|l|}
\hline & Sequence & Sensitivity & Specificity & PPV & NPV & Accuracy \\
\hline \multirow{3}{*}{ Internal opening } & T2W TSE & 96.6 & 92.6 & 99.5 & 65.8 & 96.4 \\
\cline { 2 - 7 } & $\begin{array}{l}\text { Postcontrast FS } \\
\text { T1W TSE }\end{array}$ & 98.4 & 81.5 & 98.7 & 78.6 & 97.3 \\
\hline \multirow{3}{*}{ Abscess } & T2W TSE & not calculated & not calculated & not calculated & not calculated & not calculated \\
\cline { 2 - 7 } & $\begin{array}{l}\text { Postcontrast FS } \\
\text { T1W TSE }\end{array}$ & 100 & 100 & 100 & 100 & 100 \\
\hline \multirow{2}{*}{ Secondary tract } & T2W TSE & 96.7 & 99.2 & 98.3 & 98.5 & 98.5 \\
\cline { 2 - 7 } & $\begin{array}{l}\text { Postcontrast FS } \\
\text { T1W TSE }\end{array}$ & 98.4 & 97.7 & 95.3 & 99.2 & 97.9 \\
\hline
\end{tabular}

Table 6. Comparison between T2W TSE and post-contrast FS T1W TSE sequences in the characterisation of fistulas.

MRI correctly identified 381/385 (99.0\%) internal openings. In one case, MRI failed to detect the presence of internal openings, and in 3 other cases, there was a mismatch between MRI and surgical findings.

Abscess. 47 abscesses were detected in $41(11.2 \%)$ patients. Abscess locations were as follow: 16 (34\%) in ischioanal, $16(34 \%)$ in intersphincteric, $10(21.3 \%)$ in perianal, $4(8.5 \%)$ in supralevator and $1(2.1 \%)$ in deep postanal space. 15/41 (36.6\%) patients had horseshoe abscess development.

The mean diameter of abscesses was $2.3 \pm 1.1 \mathrm{~cm}$. Both sensitivity and specificity of MRI in diagnosing abscesses were $100 \%$.

Secondary tract. 132 secondary tracts were detected on MRI in 101 (27.5\%) patients. 78 (77.2\%) patients had one secondary tract. $23(22.8 \%)$ patients had multiple secondary tracts: $18(17.8 \%)$ had two, $3(3.0 \%)$ had three, $1(1.0 \%)$ had four and $1(1.0 \%)$ had five secondary tracts. The agreement between MRI and surgical findings in identifying the location of secondary tracts is shown in Table 5.

The sensitivity, specificity, PPV, NPV and accuracy of T2W TSE and post-contrast FS T1W TSE sequences to detect internal openings, secondary tracts and abscesses are shown in Table 6.

\section{Discussion}

Our study has demonstrated that perianal fistula occurs predominantly in adult males with the male to female ratio of 9:1 and the mean age of disease is 40 years, which are consistent with previous reports $\mathrm{s}^{3,15,17,18}$.

We observed a strong association between the distance from external opening to anal verge and the type of anal fistula. The majority of intersphincteric (95.7\%) and low transsphincteric (71.8\%) fistulas had their external openings near the anal verge $(\leq 3 \mathrm{~cm}$ ) (Fig. 1$)$, while external openings were located more distally to the anal verge $(>3 \mathrm{~cm})$ for the most of transsphincteric $(90.7 \%)$, and all the suprasphincteric $(100 \%)$ and extrasphincteric (100\%) fistulas. However, there were some exceptional cases, especially when external openings on the posterior midline of scrotum. In these cases, external openings were distal to the anal verge, but fistulas might still be low-transsphincteric due to their long subcutaneous course.

MRI has been studied by many researchers and it is now recognised as an essential imaging modality in the preoperative evaluation and management of perianal fistulas. In particularly, MRI has the ability to detail accurately fistulas, associated abscesses and secondary extensions, which would otherwise be challenging for other radiology modalities. In addition, it can provide comprehensive images of the anatomical correlation between fistulas and anal sphincters, pelvic floor and levator ani muscle. This information is very important in surgical planning to remove completely infected lesions and reduce complications as well as recurrence. One previous study has demonstrated that the recurrence rate reduces substantially $(\sim 75 \%)$ following MRI navigated surgery ${ }^{5}$. 

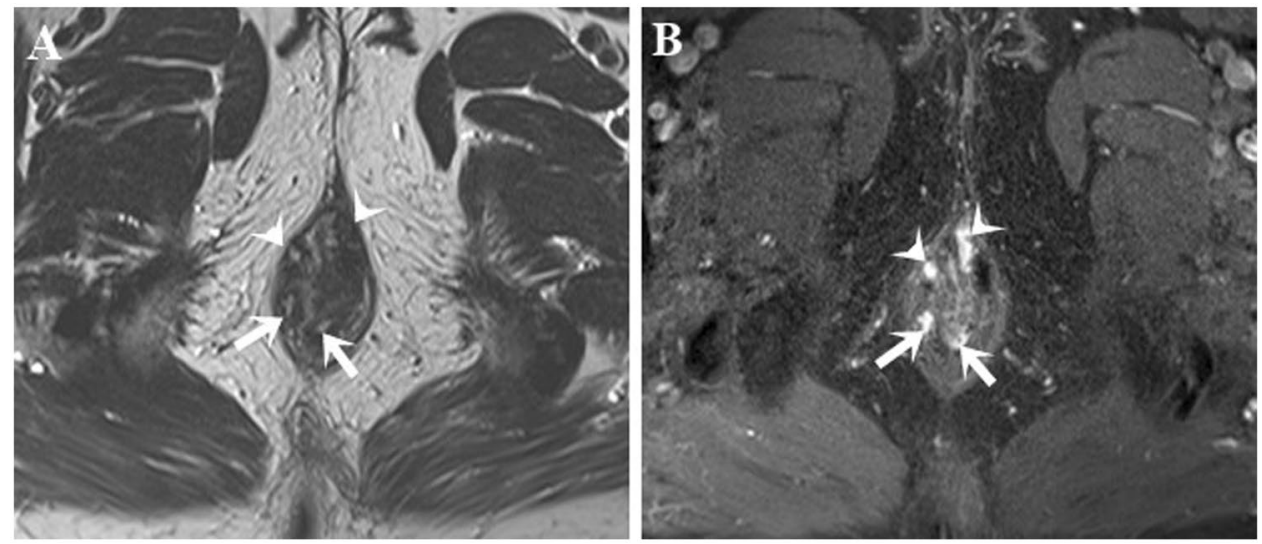

Figure 1. Intersphincteric and transsphincteric fistulas. Axial T2W TSE (A) and post-contrast FS T1W TSE (B) images show four fistulas in one patient: two intersphincteric fistulas (arrow) and two low transsphincteric fistulas penetrating the subcutaneous part of external anal sphincter (head arrow).

Consistent with the previous study by Buchanan et al. ${ }^{8}$, the majority of 411 primary tracts found during surgery were transsphincteric (64.0\%) and intersphincteric (22.4\%). These rates differ from the previous report that the most common fistulas are the intersphincteric ${ }^{3,13}$. However, this could be explained by our different study population which included only patients who had preoperative MRI prior to corrective surgery. In contrast to our study, other authors ${ }^{3,13}$ included those who had corrective surgery with or without preoperative MRI. Most of the patients in our study had a single primary tract $(90.7 \%)$, which was similar to the previous study by Baik et al. $(83 \%)^{19}$.

The strong agreement observed in our study $(k=0.89)$ between MRI and surgical findings in terms of the classification of primary tracts is very close to a previous study $(\mathrm{k}=0.84)^{8}$. Similarly, Singh et al. ${ }^{15}$ and Beets-Tan et al. ${ }^{20}$ reported that MRI had a high accuracy rate ( $94 \%$ and $93 \%$ respectively) in detecting primary tracts. Our study, as well as previous studies, support the concept that MRI is the imaging modality of choice in the preoperative assessment of anal fistulas ${ }^{8,15,16}$. In our study, MRI was found to misclassify $10 \%$ of primary tracts compared with the reference standard. However, this misclassification rate could be attributed to a minor difference in the tract description between radiologists and surgeons. In particular, tracts crossing the most distal fibres of subcutaneous external sphincter were identified as intersphincteric fistulas on MRI, and then reassigned as transsphincteric fistulas by surgeons.

Precise evaluation of the location of internal openings is essential for a successful surgical outcome. On MRI, an internal opening is defined as the nearest point of a fistula to the anal canal, which is often seen in the intersphincteric space and it is nearly impossible to trace along a tract to its very end into the anal mucosa. Previous authors have suggested that the potential location of internal opening, in most cases, is often positioned in the most inflammatory area of intersphincteric space ${ }^{5,20}$. In our study, we used the similar strategy and noticed that the sensitivity, specificity, PPV, NPV and accuracy of MRI for depicting the internal opening were $99 \%, 85.2 \%, 99 \%, 85.2 \%, 98 \%$, respectively. These findings are not surprising and in agreement with previous studies. Comparable high rates were reported by Beets-Tan et al. ${ }^{20}$ (sensitivity, specificity, PPV and NPV - 96\%, 90\%, 90\% and 96\% respectively) and Singh et al. ${ }^{15}$ (sensitivity, specificity and PPV $-96 \%, 80 \%$, and $98 \%$ respectively). In another study ${ }^{18}$, the concordance rate for the identification of internal openings between MRI and surgery was $87 \%$. The majority of internal openings were observed at the dentate line where most anal glands empty into the anus.

MRI has the advantages of resolution and large field of view, especially when a multichannel phased array coil is combined with a high field strength of 1.5-Tesla or 3-Tesla. In this study, we used the diameter-based criteria, proposed by Torkzad et al. ${ }^{16}$, to classify fluid collections, i.e., a roundish fluid collection $\geq 10 \mathrm{~mm}$ was identified as an abscess while an elongated fluid collection $<10 \mathrm{~mm}$ was identified as a fistula (Fig. 2). We have found that both sensitivity and specificity of MRI in differentiating abscesses from fistulas were $100 \%$. Prior to our study, Beets-Tan et al. ${ }^{20}$ and Singh et al. ${ }^{15}$ also reported high sensitivity ( $96 \%$ and $87.5 \%$ respectively) and high specificity ( $97 \%$ and $95.2 \%$ respectively) with regards to abscess detection.

The agreement between MRI and surgical findings in terms of identifying the location of secondary tracts was found to be good $(\mathrm{k}=0.94)$ (Fig. 3), approximating to the previous study by Buchanan et al. $(\mathrm{k}=0.88)^{8}$. There were 11 false-positive secondary tracts, identified as tiny or short fistulas on MRI. We suspect that these tracts were probably resected together with primary tracts, however, not detected by surgeons.

Both T2W TSE and post-contrast FS T1W TSE sequences demonstrated a high degree of accuracy in depicting the characteristics of fistula. In contrast to a ring-enhancing abscess, homogeneous enhancement is often seen in active inflammation on post-contrast FS T1W sequence (Fig. 4). Therefore, post-contrast FS T1W TSE sequence is suited better for distinguishing between an abscess and inflammation as they both appear hyperintense on T2W TSE sequences. However, T2W TSE sequences tended to be more optimal to position fistulas in their anatomical plane and assess their connection to anal sphincters. Torkzad et al. ${ }^{16}$ have indicated that T2W sequences must be included in the MRI protocol for the most reliable evaluation of fistula-in-ano in conjunction with post-contrast T1W sequences. Conversely, Singh et al. ${ }^{15}$ concluded that both T2W TSE and post-contrast 

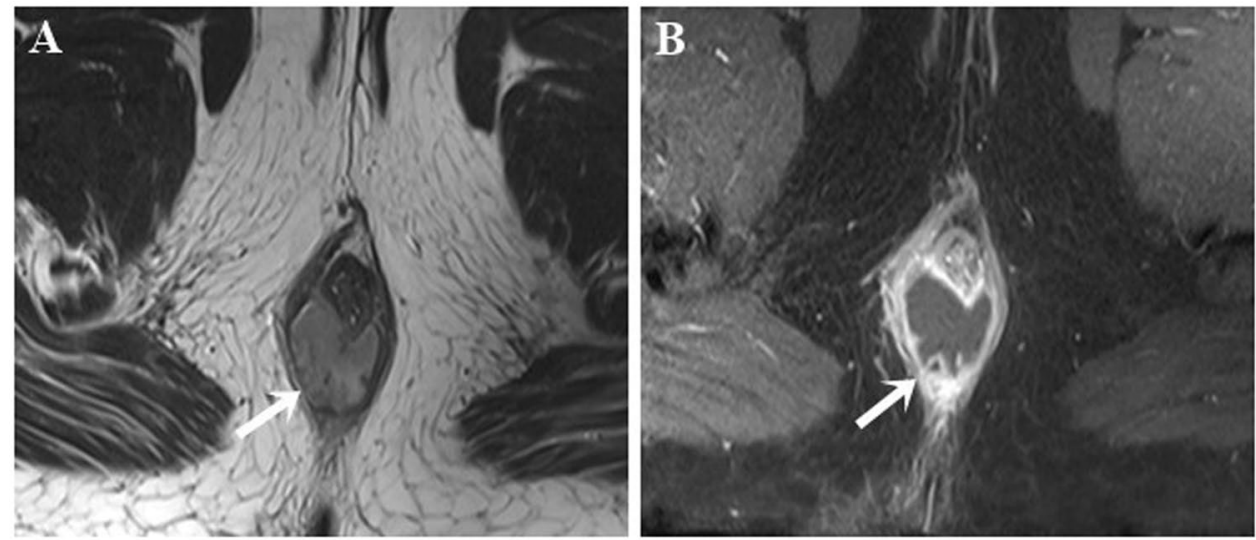

Figure 2. Intersphincteric horseshoe abscess. Axial T2W TSE (A) and post-contrast FS T1W TSE (B) images show a horseshoe abscess (arrow) with high signal intensity on T2W TSE and rim enhancement on post-contrast FS T1W TSE.
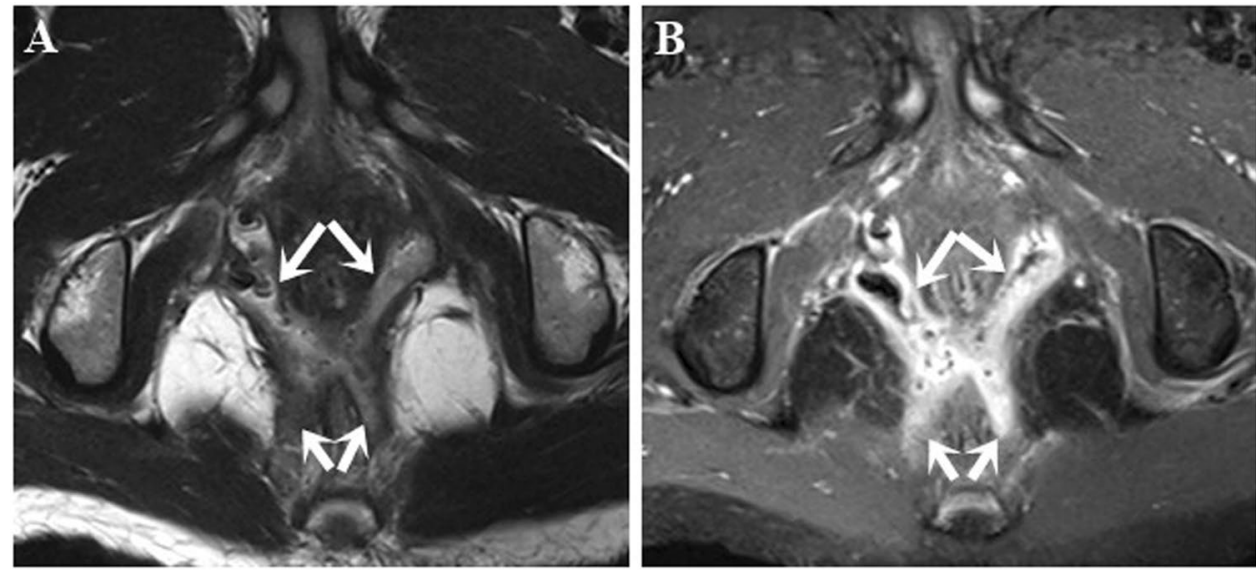

Figure 3. Secondary tracts. Axial T2W TSE (A) and post-contrast FS T1W TSE (B) images show a high transsphincteric fistula with secondary tracts in the ischioanal space (arrows).
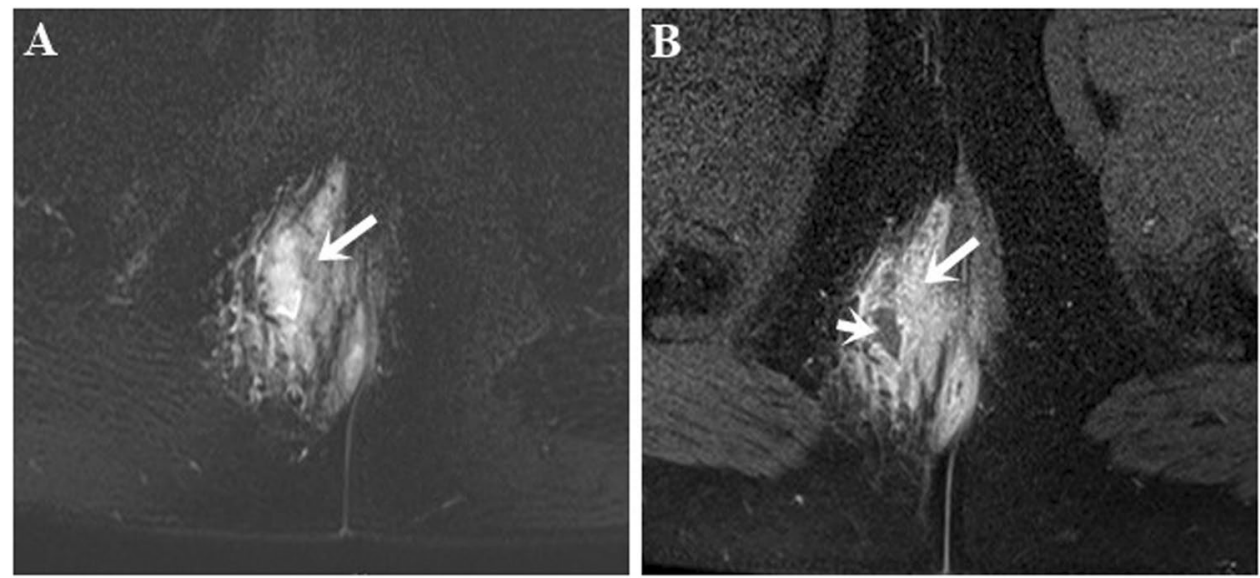

Figure 4. Abscess and active inflammation. Axial FS T2W TSE image (A) shows a hyperintense lesion which may be an abscess and/or active inflammation. Axial post-contrast FS T1W TSE image (B) differentiates an abscess (short arrow) from adjacent active inflammation (long arrow). 
FS T1W TSE sequences were comparable for assessing abscess and horseshoeing. However, a limitation of this study was that the study population did not include patients who had recurrent fistulas and/or previous surgery.

In interpreting our observations, specific limitations should be acknowledged: our study was retrospective in design; and all surgeons, albeit proctologists, had no equivalent level of experience in assessing fistula-in-ano, which might compromise the quality and consistency of the reference standard.

\section{Conclusions}

Our study reinforces the importance of MRI in the characterisation and preoperative mapping of fistula-in-ano which are major contributors to the surgical prognosis. Using both T2W TSE and post-contrast FS T1W TSE sequences is a necessity for an adequate assessment of fistula-in-ano and contrast study should be included to differentiate an abscess from active inflammation.

Received: 10 May 2019; Accepted: 13 November 2019;

Published online: 29 November 2019

\section{References}

1. de Miguel Criado, J. et al. MR imaging evaluation of perianal fistulas: spectrum of imaging features. Radiographics: a review publication of the Radiological Society of North America, Inc 32, 175-194, https://doi.org/10.1148/rg.321115040 (2012).

2. Nguyen, D. H. Gastrointestinal surgical treatment (ed. Nguyen, D. H.) 191-217 (Medical Publishing House, 2007).

3. Abcarian, H. Anal fistula: principles and management. (ed. Abcarian, H.) 13-32 (Springer, 2014).

4. Do, D. C. The reasons of failure in surgery for anal fistula. Ho Chi Minh City Medical Journal 11, 177-179 (2007).

5. Halligan, S. \& Stoker, J. Imaging of fistula in ano. Radiology 239, 18-33, https://doi.org/10.1148/radiol.2391041043 (2006).

6. Lilius, H. G. Fistula-in-ano, an investigation of human foetal anal ducts and intramuscular glands and a clinical study of 150 patients. Acta chirurgica Scandinavica. Supplementum 383, 7-88 (1968).

7. Kuijpers, H. C. \& Schulpen, T. Fistulography for fistula-in-ano. Is it useful? Diseases of the colon and rectum 28, 103-104, https://doi. org/10.1007/bf02552656 (1985).

8. Buchanan, G. N. et al. Clinical examination, endosonography, and MR imaging in preoperative assessment of fistula in ano: comparison with outcome-based reference standard. Radiology 233, 674-681, https://doi.org/10.1148/radiol.2333031724 (2004).

9. Vo, T. D. \& Nguyen, Q. T. D. Endorectal ultrasonography with hydrogen peroxide in diagnosis of anal fistulas and ano-vaginal fistulas. Ho Chi Minh City Medical Journal 11, 17-23 (2007).

10. Agha, M. E., Eid, M., Mansy, H., Matarawy, K. \& Wally, M. Preoperative MRI of perianal fistula: Is it really indispensable? Can it be deceptive? Alexandria Journal of Medicine 49, 133-144, https://doi.org/10.1016/j.ajme.2012.09.003 (2013).

11. Chaudhari, N. H., Sinkar, A. D. \& Swoyam, S. Role of magnetic resonance imaging in evaluation of perianal fistulas. International Journal of Research in Medical Sciences 4, 482 (2016).

12. Konan, A., Onur, M. R. \& Özmen, M. N. The contribution of preoperative MRI to the surgical management of anal fistulas. Diagnostic and Interventional Radiology 24, 321 (2018).

13. Parks, A., Gordon, P. H. \& Hardcastle, J. A classification of fistula-in-ano. British Journal of Surgery 63, 1-12 (1976).

14. Morris, J., Spencer, J. A. \& Ambrose, N. S. MR imaging classification of perianal fistulas and its implications for patient management. Radiographics: a review publication of the Radiological Society of North America, Inc 20, 623-635 (2000).

15. Singh, K., Singh, N., Thukral, C., Singh, K. P. \& Bhalla, V. Magnetic resonance imaging (MRI) evaluation of perianal fistulae with surgical correlation. Journal of clinical and diagnostic research: JCDR 8, RC01 (2014).

16. Torkzad, M. R., Ahlström, H. \& Karlbom, U. Comparison of different magnetic resonance imaging sequences for assessment of fistula-in-ano. World journal of radiology 6, 203 (2014).

17. Buchanan, G. et al. Effect of MRI on clinical outcome of recurrent fistula-in-ano. The Lancet 360, 1661-1662 (2002).

18. Le, T. D. \& Vo, T. D. Initial investigation of the value of MRI in anal fistula. Ho Chi Minh City Medical Journal 14, 87-91 (2010).

19. Baik, J., Kim, S. H., Lee, Y. \& Yoon, J.-H. Comparison of T2-weighted imaging, diffusion-weighted imaging and contrast-enhanced T1-weighted MR imaging for evaluating perianal fistulas. Clinical imaging 44, 16-21 (2017).

20. Beets-Tan, R. G. et al. Preoperative MR imaging of anal fistulas: does it really help the surgeon? Radiology 218, 75-84 (2001).

\section{Acknowledgements}

The authors thank Dr. Thai Thanh Truc from the Department of Statistics, University Medical Centre, for assistance with the statistical analyses. This research received no specific grant from any funding agency.

\section{Author contributions}

D.V.: designed and conducted research, analysed data, wrote and reviewed paper; C.P.: conducted research and analysed data; L.N.: designed research, analysed data and reviewed paper; H.L.: conducted research and reviewed paper; T.N.: reviewed paper; H.P.: reviewed paper and has primary responsibility for the final version of manuscript. All authors read and approved the manuscript.

\section{Competing interests}

The authors declare no competing interests.

\section{Additional information}

Correspondence and requests for materials should be addressed to D.V.

Reprints and permissions information is available at www.nature.com/reprints.

Publisher's note Springer Nature remains neutral with regard to jurisdictional claims in published maps and institutional affiliations. 
(c) (i) Open Access This article is licensed under a Creative Commons Attribution 4.0 International License, which permits use, sharing, adaptation, distribution and reproduction in any medium or format, as long as you give appropriate credit to the original author(s) and the source, provide a link to the Creative Commons license, and indicate if changes were made. The images or other third party material in this article are included in the article's Creative Commons license, unless indicated otherwise in a credit line to the material. If material is not included in the article's Creative Commons license and your intended use is not permitted by statutory regulation or exceeds the permitted use, you will need to obtain permission directly from the copyright holder. To view a copy of this license, visit http://creativecommons.org/licenses/by/4.0/.

(C) The Author(s) 2019 